Research Article

\title{
Food for thought: change in ego-dystonicity and fear of self in bulimia nervosa over the course of inference based treatment
}

\begin{abstract}
Degree of ego-dystonicity in obsessions is clinically relevant to the conceptualization and treatment of eating disorders (EDs). Obsessive-Compulsive Disorder (OCD) research has suggested that the transformation of intrusive thoughts into obsessions is linked to the degree to which intrusive thoughts threaten core perceptions of the self. Given the recognized overlap between EDs, particularly anorexia and bulimia nervosa (BN), and OCD in phenomenology and psychological characteristics, a 24-week cognitive inferencebased therapy (IBT) program shown to be effective in treating OCD was adapted to treat EDs. This study explores the change in

a. ED symptom severity,

b. The degree of ego-dystonicity in obsessions, and

c. Fear of self, motivational, mood and anxiety measures from pre-IBT to six-month follow-up. Changes in ego-dystonicity, fear of self, ED symptoms and motivational stage were assessed in 15 women with BN over the course of IBT and at follow-up. Clinical implications are discussed.
\end{abstract}

Keywords: ego-dystonicity, bulimia nervosa, cognitive therapy, fear of self, inferencebased therapy
Volume 3 Issue 3 - 2015

\author{
Magali Purcell Lalonde, ${ }^{1,2}$ Kieron O \\ Connor ${ }^{1,2}$ \\ 'Montreal Mental Health University Institute, Canada \\ 2University of Montreal, Canada
}

Correspondence: Kieron O'Connor or Magali Purcell Lalonde, Montreal Mental Health University Institute, 733। Hochelaga street, Montreal (Quebec), Canada, HIN 3V2, Canada, Email maali.purcell.lalonde@umontereal.ca

Received: August 07,20I5| Published: August 07, 2015

\section{Key practitioner message}

a. In the IBT conception of obsessions as inferences of doubt, BN possesses clear obsessional-compulsive characteristics where doubt is also the source of distress.

b. This study suggests that a cognitive inference-based therapy can be effectively used to treat $\mathrm{BN}$ by decreasing ego-syntonic obsessions, strong overvalued ideas, and fear of self via a focus on self-cognitions and reasoning about self.

c. In the current study, a significant improvement in both ED obsessions and compulsive behaviour was observed over the course of treatment and persisted at six-month follow-up. Indeed, $80 \%$ of the sample demonstrated a clinically significant reduction in ED symptoms.

\section{Introduction}

A distinguishing feature of obsessions is their ego-dystonicity. ${ }^{1}$ Purdon et al., ${ }^{2}$ authors of the Ego Dystonicity Questionnaire (EDQ), define an ego-dystonic thought as: 'One that is perceived as having little or no context within one's own sense of self or personality. '-The thought gives rise to considerable emotional distress and is resisted.' (p. 200). In contrast, ego-syntonic obsessions are consistent with an individual's self-image, values and goals. Recent research has shown that ego-dystonicity and ego-syntonicity characterize obsessions in eating disorders (EDs) and that these factors are clinically relevant to the conceptualization and treatment of Eds. ${ }^{3}$ A greater degree of ego-syntonicity in obsessions of individuals with obsessive-compulsive disorder (OCD) has been associated with treatment resistance, treatment refusal, poor insight, and is often typical in individuals with overvalued ideas (OVI) or a high degree of conviction towards obsessional doubts. ${ }^{4,5}$ If the content of an obsession is perceived as consistent with a person's values, then the investment in resisting it may not be as great; thus may weaken motivation and compliance to treatment. ${ }^{6,7}$

Although obsessions in patients suffering from EDs have often been described as ego-syntonic, Roncero et al., ${ }^{8}$ demonstrated that intrusive thoughts experienced by ED patients can simultaneously be both ego-dystonic and ego-syntonic: For example one patient stated: "All the time, no matter what I do, thoughts like these bother me: you're fat, you have to vomit, you ate too much. I can't stand it. I can't concentrate on anything. But for me it is so important to have a good physique, that I find it normal and rational to have thoughts like this. So I will not fight the thoughts, I'll do what they say." (p. 72).

Mazure CM et al., ${ }^{9}$ found that although more than half of their 40 ED sample of ED patients rated their eating-related preoccupations as ego-syntonic, 10 rated them as ego-dystonic. Similar findings were replicated in recovered ED patients and restrained-eating control subjects. ${ }^{10}$ The extent of ego-dystonicity varies between individuals with EDs. In particular, many authors suggest that the transformation of intrusive thoughts into obsessions is linked to the degree to which intrusive thoughts threaten core perceptions of the self., ${ }^{2,311}$

OCD researchers have proposed that self-themes and the areas of life where an individual has important self doubts render a person vulnerable to a particular selective set of obsessional doubts. ${ }^{12,13}$ Aardema \& $\mathrm{O}^{\prime}$ Connor $^{12}$ have studied the threatening character of obsessions that arise from within the individual. In individuals suffering from OCD, the fear of who they could become, seems a key self threat. ${ }^{12}$ Rachman $\mathrm{S}^{14}$ also emphasized that individuals with obsessional disorders believe deep down that they have unacceptable aspects to their identity. Markus \& Nurius ${ }^{15}$ define the feared self as a set of attributes that people are worried of becoming and thus constantly striving not to become. Ogilvie $\mathrm{DM}^{16}$ argues that the feared self contains awful memories, undesired emotions, frightening events, and socially unac- 
ceptable thoughts or behaviour. Similarly, Ferrier \& Brewin ${ }^{17}$ have reported evidence for the existence of the concept of the "fear of self" in OCD as they found that those with OCD make more negative inferences about themselves on the basis of their intrusions.

Recent research has demonstrated that ED patients have unconditional negative representations of the self or core beliefs that are not directly related to food, weight or shape and different patterns of these self-beliefs are associated with binge-eating and vomiting behaviours. ${ }^{18-20}$ In EDs, the self-theme is frequently a feared identity of what the person could become (e.g., "I could become someone fat and unlovable"). That is, individuals with EDs are convinced that they could become a person they fear becoming if they do not take precautions. They demonstrate a strong investment in their sense of feared self or "self-as-could-be", at the expense of their actual self or sense of "self-as-is". This results in a skewed experience of self that is not based on reality. ${ }^{12}$ Among other fears, individuals with EDs may fear becoming fat, out of control, lazy, unattractive, and unloved.

Purcell Lalonde et al., ${ }^{3}$ have found that in EDs, ego-dystonicity in obsessions is associated to the concept of fear of self. There is a recognized overlap between EDs, particularly anorexia nervosa (AN) and bulimia nervosa (BN), and OCD in terms of diagnosis, phenomenology, epidemiology, comorbidity, and psychological characteristics related to the disorder. ${ }^{21}$

\section{Treatment}

Cognitive-behavioural therapy (CBT) is the current treatment of choice for $\mathrm{BN}^{22}$ and effective for the treatment of $\mathrm{AN}$, although not shown as superior to other treatments. ${ }^{23}$ Although CBT can be effective for OCD and Eds, ${ }^{22}$ approximately $50 \%$ of individuals with an $\mathrm{ED}^{24}$ and up to $40 \%$ with $\mathrm{OCD}^{25}$ refuse or abandon $\mathrm{CBT}$. In the case of those with $\mathrm{BN}$, more than half fail to reach asymptomatic criteria at follow-up ${ }^{26,23}$ and about a quarter drop out of treatment. ${ }^{27}$

EDs are associated with overvalued ideas (OVI) firmly held beliefs towards obsessional doubts. Irrational thoughts such as "If I feel full, I'm gaining weight" and "If I eat food that I usually deny myself, I'll lose control" are representative of OVI held by individuals with Eds. ${ }^{28}$ Specialized CBT for individuals with OVI may require a more thorough investigation of cognitive maintaining factors prior to the introduction of behavioural exercises..$^{29}$

Inference-Based Therapy (IBT) was developed by O' Connor et al., ${ }^{13}$ to treat individuals with OCD with particularly strong OVI. Studies have demonstrated that IBT is equally effective in individuals with OVI and individuals without OVI. ${ }^{30-32}$ IBT has been adapted to treat individuals with Eds. ${ }^{33}$ The reasoning process hypothesized to be common to OCD and EDs and to lead to obsessions is termed inferential confusion, which is defined as a tendency as a confusion between reality and possibility, where the person distrusts the senses, and invests in remote and often imaginary possibilities at the expense of reality. Inferential confusion was found to be associated with treatment outcome in an OCD sample receiving $\mathrm{CBT}^{34}$ and another receiving $\mathrm{IBT}^{35}$

According to the IBT model, the obsessional sequence begins with a doubt (e.g., "May be I gained weight") generated by a subjective reasoning narrative and is a primary treatment target. This obsessional doubt serves as the basis for subsequent obsessions and distress, and generates concerns about anticipated consequences (e.g., "If I gain weight, I'll be fat and will be rejected by others"). The aim of IBT is to decrease the person's degree of conviction regarding obsessions in a non-confrontational manner rather than challenging beliefs, modify the reasoning narrative producing the doubt, return the person to the world of common sense perception, and so have a positive impact on the rest of the obsessional sequence in $\mathrm{ED}$, including imagined consequences, anxiety, and compulsive behaviour. Additionally, IBT addresses doubts about the self and unconditional negative representations of the self, present in EDs.

Since individuals with EDs often have OVI and IBT has been shown effective in decreasing OVI, we adapted and applied IBT to EDs. In the present study, our first aim was to investigate if ED symptoms decrease following IBT. Second, we examine our hypothesis that ego-dystonicity in obsessions and compulsions as well as OVI decrease over the course of IBT. Third, we explore the change in cognitive process measures including inferential confusion, feared self and obsessive beliefs following IBT.

\section{Method}

\section{Participants}

Participants were recruited through advertising at University of Montreal and University of Quebec in Montreal as well as through ANEB Quebec, a non-profit organization assisting individuals suffering directly or indirectly from an ED. Forty three individuals contacted the Montreal Mental Health University Institute offering an outpatient 24-week intervention program for AN and BN. Participants included in the study were adults with a principal diagnosis of AN (AN-restrictive (r) or binge-purge subtype (bp)) or BN (BN-purging subtype (p) or non-purging subtype (np)) in accordance with criteria put forth in the Diagnostic and Statistical Manual of Mental Disorders, 4th edition, text revision (DSM-IV-TR). ${ }^{1}$ Amenorrhea was not a requirement for individuals diagnosed with $\mathrm{AN}$, given recommendations that this criterion be removed from the core diagnostic features in DSM V. ${ }^{36}$ All medications were stabilized 3months prior to participation.

\section{Assessment}

Assessment included a two-stage process involving telephone screening interviews followed by initial questionnaires returned by mail and a face-to-face diagnostic interview with a psychology doctoral student (supervised by a psychologist) using the Structured Clinical Interview for DSM-IV Clinical Version $\left(S C I D-I / C V ; .{ }^{37} \mathrm{~A}\right.$ licensed psychologist then confirmed the primary diagnosis of $\mathrm{AN}$ or $\mathrm{BN}$.

\section{Measures}

Participants completed a battery of questionnaires before the start of therapy (pre-treatment) as well as at mid, post-treatment, and at six-month follow-up. Their ED symptoms were also assessed by a doctoral psychology student in a face-to-face interview at mid, post, and at six-month follow-up.

\section{Clinician assessment}

Eating disorder examination ${ }^{18}$ : The EDE is a semistructured interview composed of four subscales of disordered eating including restraint, concern with eating, concern with weight, and concern with shape. It provides frequency and severity ratings for key behavioural and attitudinal aspects of EDs that can assess the presence of an ED according to DSM-IV criteria. Response scales range from 0 (no pathology) to 6 (extreme pathology). The subscales of the EDE have good psychometric properties with internal consistency reliability coefficients ranging from $\alpha=0.67$ to $0.90 .{ }^{38}$ 
Overvalued ideas scale ${ }^{5}$ : The OVIS is an 11-item semistructured interview assessing different dimensions of overvalued ideas such as fixidity (whether the belief persists), effectiveness of compulsions (in reducing feared outcome probability), reasons others do not share the belief (others are unaware or misinformed). The OVIS has good psychometric properties with an internal consistency reliability coefficient of $\alpha=0.88$, test-retest reliability of $r=0.86$ and interrater reliability of $r=0.88$.

Structured clinical interview for $\mathbf{d s m}$-iv clinical version ${ }^{37}$ : The SCID-I/CV is the semistructured interview of choice to establish a differential diagnosis of axis I disorders according to the DSM-IV. It has good psychometric properties. ${ }^{39}$

Yale-brown-cornell eating disorder scale ${ }^{9}$ : The YBC-EDS is a semistructured interview assessing symptom severity and cognitive as well as behavioural changes pertaining to ED obsessions and compulsions. In addition, a subscale evaluates the degree of ego-syntonicity of obsessions and compulsions and another estimates motivation to change. The YBC-EDS is an adaptation of the Yale-Brown Obsessive Compulsive Scale (Y-BOCS) ${ }^{40,41}$ used to assess OCD symptoms and severity. The subscales of the instrument have good psychometric properties with internal consistency reliability coefficients of $\alpha=0.87$ to 0.90 and interrater reliability coefficients of $\alpha=0.81$ to 0.99 .

\section{Self-report symptom measures}

Ego dystonicity questionnaire ${ }^{2}$ : The EDQ is a 37 -item questionnaire that measures the degree of ego-dystonicity in obsessions according to four key concepts: inconsistency with morals, repugnance, implications of thought for personality, and irrationality. The EDQ has demonstrated adequate internal consistency reliability ( $\alpha=0.76$ to 0.89 ) and evidence of satisfactory construct validity, with significant relationships with obsessive-compulsive symptoms, mood and appraisal of obsessions. ${ }^{2}$ The EDQ version used in the present study was modified to prompt individuals that the questionnaire was focusing on obsessions about food, weight, and/or shape.

Fear of self questionnaire ${ }^{42}$ : The FSQ is a 20 -item questionnaire that measures the degree of investment in an individual's "sense of self-as could-be" or feared self in contrast to their "sense of self-as-is", a concept related to ego-dystonicity. The FSQ has shown strong internal consistency $(\alpha=0.96)$, good divergent and convergent validity, including strong relationships to obsessional symptoms.

Eating disorder inventory- ${ }^{43}$ : The EDI-2 is a 91 -item questionnaire assessing different attitudes, emotions and behaviours related to disordered eating containing 11 subscales: drive for thinness, bulimia, body dissatisfaction, ineffectiveness, perfectionism, interpersonal distrust, interoceptive awareness, maturity fears, asceticism (provisional), impulse regulation (provisional), social insecurity (provisional). It is widely used to measure treatment response in ED studies. The EDI-2 has good psychometric properties with internal consistency reliability coefficients ranging from $\alpha=0.83$ to $0.93^{44}$ and test-retest reliability coefficients ranging from of $r=0.79$ to $0.95 .{ }^{45}$

Motivational stages of change questionnaire ("Questionnaire de motivation et les stades de changement") is a French self-report questionnaire designed to assess readiness to change based on ${ }^{46}$ six stages of change model and inspired from the Motivational stages of change for adolescents recovering from an eating disorder (MSCARED) questionnaire: ${ }^{47}$ (1) pre-contemplation, (2) contemplation, (3) preparation, (4) action, (5) maintenance, (6) recovery. This instrument determined in which stage of change participants described themselves currently in relation to their ED as well as their readiness to change.
Beck anxiety inventory ${ }^{48}$ : The BAI a 21 -item anxiety symptom checklist rating symptom intensity for the last week. The French translation of the BAI has adequate psychometric properties with an internal consistency reliability coefficient of $\alpha=0.84$ and a test-retest reliability of $r=0.63 .^{49}$

Beck depression inventory-II ${ }^{50}$ : The BDI-II is a 21 -item questionnaire that measures emotional, cognitive, motivational, and physiological symptoms of depression. The French version of the BDI-II has excellent psychometric properties with internal consistency reliability coefficients of $\alpha=0.92-.93$ and test-retest reliability of $r=0.93 .{ }^{51}$

Personality Disorders Questionnaire-4+ ${ }^{52}$ : The PDQ-4+ is a 99item self-report questionnaire with a true-false format that evaluates the presence of personality disorders consistent with the DSM-IV. ${ }^{1}$ The questionnaire also includes validity scales designed to assess invalid responding patterns. It has acceptable psychometric properties. For the purpose of the present study, the PDQ-4+ was used to identify predominant personality traits rather than as a personality disorder diagnostic tool, as personality disorders were not part of the exclusion criteria for participation in the study.

The Obsessional Belief Questionnaire ${ }^{53}$ : The OBQ-44 is a 44-item questionnaire that assesses four domains of obsessive beliefs including inflated responsibility for harm, overestimation of threat, intolerance of uncertainty/perfectionism, and over-importance/control of thoughts. The OBQ-44 demonstrated good internal consistency and criterion-related validity ( $\alpha=0.95$ ) in clinical and non-clinical samples. Initial validation of the French version of the OBQ-44: "Questionnaire sur les croyances obsessionnelles" 54 indicates good convergent and construct validity ( $\alpha=0.89$ to 0.93 ) and evidence of good internal consistency reliability.

Inferential Confusion Questionnaire-Expanded Version (ICQ$\mathbf{- E V}$; $^{55}$ : The ICQ-EV is a 30 -item questionnaire measuring inferential confusion. Scores range from 30 to 180 with higher scores signifying greater inferential confusion. The ICQ-EV has excellent psychometric properties with an internal consistency reliability coefficient of $\alpha=$ 0.90 and construct validity of $\alpha=0.85$.

\section{Procedure}

\section{Inclusion and exclusion criteria}

The study excluded patients with binge eating disorder, current substance abuse, current or past schizophrenia, bipolar disorder, or those with suicidal intent. Patients with other comorbid diagnoses were included in the study as long as their primary diagnosis was AN or BN. Written informed consent was obtained from all participants, and all measures and procedures were approved by the Research Ethics Board at the Montreal Mental Health University Institute. Eight individuals were excluded and referred to appropriate mental health resources.

\section{Drop outs}

Sixteen either did not return our calls after the telephone screening interview, did not return the initial questionnaires required to complete the assessment phase and/or informed us of their lack of readiness to commit themselves to our therapy program because of limited availability in their schedule and/or ambivalence regarding change. From the four participants who dropped out of treatment within the three first months of IBT, two with a diagnosis of AN-r and AN-bp were contacted by an intensive hospital-based ED clinic in Montreal as they were on the waiting list for several months, and two with a diagnosis 
of AN-bp and BN-p reported their lack of time and motivation to pursue therapy. Two participants who dropped out of treatment reported that they were in the contemplation motivational stage according to DiClemente \& Prochaska ${ }^{46}$ stages of change and were motivated to change for themselves and for others. Two others who dropped out said that they were in the action stage, and were motivated to change only for themselves. One participant who abandoned therapy had antisocial personality traits, which is associated with difficulty in forming therapeutic alliance. No other noteworthy differences in demographic or clinical characteristics were found in participants who abandoned therapy compared with treatment completers.

\section{Final sample characteristics}

In total, twelve participants completed IBT and returned the si$\mathrm{x}$-month follow-up questionnaires, one completed IBT but did not return the follow-up questionnaires, and two had recently undergone their post-treatment assessment at the time this article was written thus did not have follow-up data. Participants $(n=15)$, had a mean age of $31.07(S D=7.90)$, and a mean BMI of $24.08(S D=4.35)$. Five $(31.3 \%)$ reported that they were in the preparation stage of change and ten $(62.5 \%)$ in the action stage. Thirteen $(81.3 \%)$ said that they wanted to change only for themselves, and two (12.5\%) for themselves and for others. Number of years suffering from an ED ranged from less than one to $26(M=11.47 ; S D=7.90)$. Nine participants $(60.0 \%)$ had a diagnosis of BN-p and six $(40.0 \%)$ had a diagnosis of BN-np. The most common personality traits among participants were borderline, depressive $(n=9 ; 60.0 \%)$, avoidant $(n=8 ; 53.3 \%)$, obsessive-compulsive $(n=5 ; 33.3 \%)$, and dependent $(n=4 ; 26.7 \%)$ traits. Two had attended high school (13.3\%), six had a college-level education $(40.0 \%)$ and seven attended university $(46.7 \%)$. Nine were single (never married; $60.0 \%$ ), four were married $(26.7 \%)$, and two were separated/divorced (13.3\%). Four had one child or more (26.7\%) and eleven did not (73.3\%). Seven were working full time (46.7\%), four were students $(26.7 \%)$, two were working part time (13.3\%), one was unemployed and one was a full-time mother (13.4\%). Three had a yearly individual income of less than $10,000 \$$, three from 10,000 to $19,999 \$$, one from 20,000 to $29,999 \$$, six from 30,000 to $39,999 \$$, and two of $40,000 \$$ or more.

\section{Treatment}

Four clinical assessment sessions were followed by 20 weekly 1-hour sessions of IBT ( 24 sessions in total) delivered by a licensed and experienced psychologist trained in IBT at the Montreal Mental Health University Institute and supervised for adherence to treatment protocol by a senior IBT therapist.

Sessions 5 to 7 were dedicated to psycho-education for EDs addressing psychological, social, and physical impacts of being underweight; the consequences of restricting food intake; the consequences of binging, vomiting, laxative and diuretic use ${ }^{56,57}$ the ineffectiveness of dieting; the role of water retention in adding weight; healthy eating habits; the role of individual metabolism in the maintenance of a natural and healthy body weight; and the non-trivial role of disordered eating thoughts and behaviours.

Subsequent therapy focused on:

a. The distinction between the feared self and the actual or authentic self;

b. The distinction between an obsessional doubt and a normal doubt;

c. ED logic; d. ED doubt as $100 \%$ irrelevant to the "here and now;"

e. Exploring the narrative supporting the doubt;

f. Crossing the barrier from reality into ED imagination;

g. Identification of reasoning errors that create and maintain the obsessional narrative;

h. Establishing the false nature of the ED doubt;

i. Establishing the selective nature of the ED doubt;

j. Identifying vulnerable self themes in obsessional narratives;

$\mathrm{k}$. Learning to trust the reality of the senses and to tolerate the void left by decreased obsessional behaviour.

\section{Results}

Data were analysed using SPSS 17.0. Non-parametric statistics were performed because of a small sample size and because data did not meet the stringent assumptions of parametric tests concerning normality and homogeneity of variance, according to guidelines set forth by ${ }^{58}$ Wilcoxon Signed Rank Tests and Friedman Tests were used after checking for specific assumptions related to these non-parametric tests. The cut-off for significance was determined a priori at $p<$ .05 as non-parametric tests tend to be less sensitive. Effect sizes were measured using Cohen $\mathrm{J}^{59}$ criteria.

\section{Longitudinal changes in ED symptoms}

The results of the Friedman Test indicated that there was a statistically significant decrease in YBC-EDS total severity scores across the four time points (pre-, mid-, post-therapy, and six-month follow-up) (see Table 1). Inspection of the median values showed a decrease in YBC-EDS scores from pre- to mid-IBT to post-IBT and a further decrease at follow-up. A significant decrease was also observed across the four time points in YBC-EDS Obsession scores as well as in YBC-EDS Compulsion scores (Table 1). A Wilcoxon Signed Rank Test (WSRT) demonstrated a significant reduction in YBC-EDS total scores following IBT (with Bonferroni corrections), with a large effect size and at six-month follow-up, with a large effect size. The median YBC-EDS total, obsessions and compulsions scores decreased from pre- to post-IBT program and further significantly decreased at follow-up, with large effect sizes (Table 1).

Although results on the Friedman Test did not show a significant difference in EDE restraint scores across the four time points, the WSRT indicated a significant reduction in this subscale scores following IBT, with a medium effect size, but not at six-month follow-up. Inspection of the median values showed a decrease in EDE restraint scores from pre- to mid-IBT to post-IBT, but a slight increase at follow-up (Table 1). A Friedman Test revealed a significant decrease in EDE eating, weight, and shape concern scores across the four time points. A WSRT indicated a significant decrease from pre- to post-IBT in median EDE eating, weight, and shape concern scores, with large effect sizes, and further significantly decreased at follow-up, with medium to large effect sizes (Table 1).

A Friedman Test showed a significant increase in participants' report of their ideal weight in the EDE, however only 8 participants answered this question across all time points. A WSRT confirmed a significant increase from pre- to post-IBT in median ideal weight, with a large effect size, and a slight decrease at follow-up, but remaining a significant increase in their ideal weight compared to pre-IBT, with a large effect size (Table 2). 
Table I Longitudinal changes in eating disorder symptoms assessed by a clinician

\begin{tabular}{|c|c|c|c|c|c|c|c|c|c|c|c|c|}
\hline & $\times 2$ & $\mathbf{p}$ & Md & Md & Md & Md & $\mathbf{z}$ & $\mathbf{p}$ & $|d|$ & $\mathbf{z}$ & $\mathbf{p}$ & $|\mathbf{d}|$ \\
\hline & & & Pre- & Mid- & Post- & Follow-up & Pre-Post & \multicolumn{5}{|c|}{ Pre-Follow-up } \\
\hline YBC-EDS Total & 28.23 & $* * *$ & 23.5 & 14 & 4 & 1.5 & $-3.4 I$ & $* * *$ & 0.62 & -3.07 & $* *$ & 0.59 \\
\hline YBC-EDS Obsessions & 29.15 & $* * *$ & 12 & 7.5 & 3.5 & 1.5 & -3.42 & $* * *$ & 0.62 & -3.07 & $* *$ & 0.59 \\
\hline YBC-EDS Compulsions & 27.65 & $* * *$ & 12 & 6.5 & 0.5 & 0 & -3.3 & $* * *$ & 0.6 & -3.06 & $* *$ & 0.59 \\
\hline $\begin{array}{l}\text { YBC-EDS Ego- } \\
\text { Syntonicity in } \\
\text { Obsessions }\end{array}$ & 14.12 & $* *$ & 1.5 & I & 0 & 0 & $-|.8|$ & 0.07 & - & -2.69 & $* *$ & 0.52 \\
\hline $\begin{array}{l}\text { YBC-EDS Ego- } \\
\text { Syntonicity in } \\
\text { Compulsions }\end{array}$ & 11.96 & $* *$ & 2 & 0.5 & 0 & 0 & -2.3 & $*$ & 0.42 & -2.84 & $* *$ & 0.55 \\
\hline \multicolumn{13}{|l|}{ EDE Restraint } \\
\hline & 3.13 & 0.37 & 2.11 & 2 & 1.72 & 1.78 & -2.41 & $*$ & 0.39 & -1.65 & 0.1 & - \\
\hline EDE Eating Concern & 18.83 & $* * *$ & 2.64 & 1.5 & 0.07 & 0.29 & -2.9 & $* *$ & 0.53 & -2.43 & $*$ & 0.47 \\
\hline EDE Weight Concern & 28.27 & $* * *$ & 3.67 & 2.92 & 1.42 & 1.83 & -3.41 & $* * *$ & 0.62 & -3.06 & $* *$ & 0.59 \\
\hline EDE Shape Concern & 26.46 & $* * *$ & 5.3 & 3.8 & 2.5 & 2.4 & -3.33 & $* * *$ & 0.61 & -3.06 & $* *$ & 0.59 \\
\hline
\end{tabular}

Note: ***significant at $p<.001 ; * * p<.01 ; * p<.05$. YBC-EDS:Yale-Brown-Cornell-Eating Disorder Scale; EDE: Eating Disorder Examination

Table 2 Longitudinal changes in Eating Disorder Inventory-2

\begin{tabular}{|c|c|c|c|c|c|c|c|c|c|c|c|c|}
\hline & $\times 2$ & $\mathbf{p}$ & Md & Md & Md & Md & $\mathbf{z}$ & $\mathbf{p}$ & $|d|$ & $\mathbf{z}$ & $\mathbf{p}$ & $|d|$ \\
\hline & & & Pre- & Mid- & Post- & Follow-up & Pre-Post & & \multicolumn{4}{|c|}{ Pre-Follow-up } \\
\hline BMI & 6.84 & 0.08 & 23.15 & 23.99 & 24.76 & 24.68 & -1.19 & 0.24 & - & -0.89 & 0.37 & - \\
\hline EDI-2 Ideal Weight & 11.77 & $* *$ & 55.91 & 59.09 & 61.36 & 60.23 & -2.58 & $* *$ & 0.5 & -2.37 & $*$ & 0.48 \\
\hline EDI-2 Drive for Thinness & 14.79 & $* *$ & 17 & 11 & 5 & 6 & -2.14 & $*$ & 0.39 & -0.85 & 0.4 & - \\
\hline EDI-2 Bulimia & 19.87 & $* * *$ & 9 & 2 & I & 3 & -2.42 & $*$ & 0.44 & -0.66 & 0.51 & - \\
\hline EDI-2 Body Dissatisfaction & 5.59 & 0.13 & 19 & 12 & II & 7 & -1.37 & 0.17 & - & -0.66 & $0.5 \mathrm{I}$ & - \\
\hline EDI-2 Ineffectiveness & 14.67 & $* *$ & 6 & 3 & I & 2 & -2.42 & $*$ & 0.44 & -0.8 & 0.43 & - \\
\hline EDI-2 Perfectionism & 7.98 & $*$ & 9 & 6 & 4 & 6 & -2.05 & $*$ & 0.37 & -0.77 & 0.44 & - \\
\hline EDI-2 Interpersonal Distrust & 3.75 & 0.29 & 2 & I & 0 & 0 & -1.87 & 0.06 & 0.34 & 0 & I & - \\
\hline EDI-2 Interoceptive Awareness & 10.37 & $*$ & 9 & 4 & 3 & 5 & -2.21 & $*$ & 0.4 & -0.57 & 0.57 & - \\
\hline EDI-2 Maturity Fears & 9.22 & $*$ & 4 & 2 & I & I & -2.14 & $*$ & 0.39 & -0.41 & 0.68 & - \\
\hline EDI-2 Asceticism & 11.87 & $* *$ & 6 & 4 & 3 & 3 & -2.28 & $*$ & 0.42 & -0.85 & 0.4 & - \\
\hline EDI-2 Social Insecurity & 12.8 & $* *$ & 7 & 3 & 2 & 2 & -2.17 & $*$ & 0.4 & -0.67 & $0.5 \mathrm{I}$ & - \\
\hline
\end{tabular}

Note: ***significant at $p<.001 ; * * p<.01 ; * p<.05$. BMI: Body Mass Index

Table 3 Longitudinal changes in ego-dystonicity, fear of self, and other symptom measures

\begin{tabular}{|c|c|c|c|c|c|c|c|c|c|c|c|c|}
\hline & $\mathbf{x 2}$ & $\mathbf{p}$ & Md & Md & Md & Md & $\mathbf{z}$ & $\mathbf{p}$ & $|d|$ & $\mathbf{z}$ & $\mathbf{p}$ & $|\mathbf{d}|$ \\
\hline & & & Pre- & Mid- & Post- & Follow-up & Pre-Post & & & Pre-F & ow-up & \\
\hline EDQ Irrationality & 8.75 & $*$ & 23 & 20 & 23 & 26 & -0.47 & 0.64 & - & -1.92 & 0.06 & 0.4 \\
\hline EDQ Inconsistency with Morals & 4.76 & 0.19 & 34 & 34 & 26 & 35 & -1.02 & 0.31 & - & -1.07 & 0.29 & - \\
\hline EDQ Repugnance & 2.23 & 0.53 & 50 & 47 & 56 & 54 & -0.76 & 0.45 & - & -1 & 0.33 & - \\
\hline $\begin{array}{l}\text { EDQ Implications in Thought for } \\
\text { Personality }\end{array}$ & 4.92 & 0.18 & 39 & 31 & 27 & 33 & -1.7 & 0.09 & - & -0.46 & 0.65 & - \\
\hline OVIS & 28.36 & $* * *$ & 60.5 & 23.5 & 17 & 10.5 & $-3.4 I$ & $* * *$ & 0.62 & -3.06 & $* *$ & 0.59 \\
\hline FSQ & 6.85 & 0.08 & 55 & 38 & 35 & 35 & -2.22 & $*$ & $0.4 I$ & -0.73 & 0.46 & - \\
\hline ICQ-EV & 8.49 & $*$ & 56 & 68 & 44 & 41 & $-1.4 \mid$ & 0.16 & - & -0.31 & 0.75 & - \\
\hline $\begin{array}{l}\text { OBQ-44 Inflated Responsibility } \\
\text { for Harm }\end{array}$ & 11.25 & $* *$ & 24 & 20 & 15 & 14 & -2.7 & $* *$ & 0.49 & -0.85 & 0.4 & - \\
\hline $\begin{array}{l}\text { OBQ-44 Overestimation of } \\
\text { Threat }\end{array}$ & 5.08 & 0.17 & 10 & 12 & 10 & 8 & $-|.6|$ & 0.11 & - & -0.71 & 0.48 & - \\
\hline $\begin{array}{l}\text { OBQ-44 Intolerance of } \\
\text { Uncertainty/ } \\
\text { Perfectionism }\end{array}$ & 15.29 & $* *$ & 71 & 64 & 49 & 42 & -3.1 & $* *$ & 0.57 & -0.85 & 0.4 & - \\
\hline $\begin{array}{l}\text { OBQ-44 Over-Importance/ } \\
\text { Control of Thoughts }\end{array}$ & $13.0 \mid$ & $* *$ & 29 & 23 & 19 & 17 & -2.45 & $*$ & 0.45 & -0.85 & 0.4 & - \\
\hline
\end{tabular}


Table Continued...

\begin{tabular}{|c|c|c|c|c|c|c|c|c|c|c|c|c|}
\hline & $\times 2$ & $\mathbf{p}$ & Md & Md & Md & Md & $\mathbf{z}$ & $\mathbf{p}$ & $|d|$ & $\mathbf{z}$ & $\mathbf{p}$ & $|d|$ \\
\hline & & & Pre- & Mid- & Post- & Follow-up & Pre-Post & & & Pre-F & ow-up & \\
\hline BDI-II & 8.34 & $*$ & 18 & 6 & 4 & 4 & -2.79 & $* *$ & 0.51 & -0.7 & $0.5 I$ & - \\
\hline BAI & 5.81 & 0.12 & 10 & 5 & 5 & 8 & -2.5 & $*$ & 0.46 & -0.77 & 0.44 & - \\
\hline Motivational Stage & 29.35 & $* * *$ & 4 & 4 & 4 & 6 & -2.92 & $* *$ & 0.53 & -3.09 & $* *$ & 0.59 \\
\hline
\end{tabular}

Note: ***significant at $p<.001 ; * * p<.01 ; * p<.05$. EDQ: Ego Dystonicity Questionnaire; FSQ: Fear of Self Questionnaire; OVIS: Overvalued Ideas Scale; OBQ44: Obsessional belief questionnaire-44; ICQ-EV: Inferential confusion questionnaire-Expanded version; BDI-II: Beck Depression Inventory-II; BAI: Beck Anxiety Inventory

Results on the Friedman Test indicated that participants' BMI did not significantly differ across four time points. Although there was a non-significant increase in median BMI scores from pre- to post-IBT, they then reverted back to their initial score at follow-up, which was in the normal range for all participants (Table 2).

A Friedman Test demonstrated a significant decrease in EDI-2 drive for thinness, bulimia, ineffectiveness, perfectionism, interoceptive awareness, maturity fears, asceticism, impulse regulation, and social insecurity scores (Table 2), across the four time points. A WSRT confirmed a significant decrease from pre- to post-IBT in these median EDI-2 subscale scores, with medium effect sizes (Table 2). A trend is observed towards decrease in EDI-2 body dissatisfaction and interpersonal distrust scores from pre- to post-IBT, with a medium effect size, and body dissatisfaction scores further decreased at follow-up. Although all other subscales scores slightly increased from post-therapy to follow-up, a WSRT indicated that this increase was not significant (drive for thinness, $z=-.41, p=.68$; bulimia, $z=$ $-1.72, p=.09$; ineffectiveness, $z=-1.53, p=.13$; perfectionism, $z=$ $-.99, p=.32$; interpersonal distrust, $z=-.70, p=.48$; interoceptive awareness, $z=-1.13, p=.26$; maturity fears, $z=-1.49, p=.14$; asceticism, $z=-.67, p=.50$; impulse regulation, $z=-1.20, p=.23$; and social insecurity scores, $z=-.71, p=.48$ ).

\section{Longitudinal changes in ego-dystonicity and OVI}

A Friedman Test revealed a significant difference in EDQ irrationality scores across the four time points, but not in other EDQ subscales scores (Table 3). A WSRT indicated a trend in an increase from pre-IBT to follow-up in the degree to which participants judged their ED thoughts as irrational, with a medium effect size (Table 3). A Friedman Test showed a significant difference in YBC-EDS degree of ego-syntonicity in obsessions as well as ego-syntonicity in compulsions across the four time points and a WSRT confirmed a significant decrease from pre-IBT to follow-up in the ego-syntonicity of obsessions and compulsions, with large effect sizes (Table 1).

A Friedman Test showed a significant decrease in OVIS scores across the four time points and a WSRT confirmed a significant decrease from pre-IBT to follow-up in OVIS scores, with a large effect size (Table 3).

\section{Longitudinal changes in cognitive process measures}

In a Friedman Test, a trend was observed in decreasing FSQ scores across the four time points and a WSRT indicated a significant decrease from pre- to post-IBT in median scores, with a medium effect size (Table 3).

A Friedman Test demonstrated a significant decrease in OBQ-44 inflated responsibility for harm, intolerance of uncertainty/perfectionism, and over-importance/control of thoughts scores, and a trend towards decrease in overestimation of threat scores across the four time points (Table 3). A WSRT indicated a significant decrease from pre- to post-IBT in all median subscale scores, with medium to large effect sizes, with the exception of overestimation of threat scores. Nevertheless, overestimation of threat scores was already low at the start of therapy and still decreased at follow-up (Table 3).

A Friedman Test indicated a significant decrease in ICQ-EV total scores across the four time points, but a WSRT revealed only a significant reduction from mid- to post-IBT median scores, with a medium effect size, not at follow-up (Table 3).

\section{Changes in depression, anxiety and motivational me- asures}

Results on a Friedman Test showed a significant decrease in BDI-II scores across the four time points (Table 3). A WSRT indicated a significant reduction in median BDI-II scores from pre- to post-IBT, with a large effect size. However, an increase in scores was observed at follow-up; a WSRT revealed that the difference between post-IBT and follow-up scores was not significant (Table 3).

Although results on the Friedman Test did not show a significant difference in BAI scores across the four time points, the WSRT indicated a significant reduction in scores following IBT, with a medium effect size, but not at six-month follow-up (Table 3). Inspection of the median values showed an increase in BAI scores at follow-up, however a WSRT revealed that the difference between post-IBT and follow-up scores was not significant (Table 3).

A Friedman Test revealed a significant change in motivational stage across the four time points within the six motivational stages. Inspection of the median values showed a change from participants' feeling of being in the action stage pre-IBT to a feeling of being in the recovery stage at follow-up. A WSRT confirmed this significant progression in participants' motivational stage from pre-IBT to follow-up, with a large effect size (Table 3).

\section{Discussion}

\section{Longitudinal changes in ED symptoms}

In the current study, a significant improvement in both ED obsessions and compulsive behaviour was observed over the course of treatment and persisted at six-month follow-up. In fact, $80 \%$ of the sample $(n=15)$ demonstrated a clinically significant reduction in ED symptoms according to Emmelkamp PMG. ${ }^{60}$ Fairburn CG et al., ${ }^{61}$ reported that 40 to $50 \%$ of treatment completers ceased binge eating and purging following CBT for BN. This study's results slightly surpass this rate, with $53 \%$ ceasing their compensatory behaviours at six-month follow-up. Participants' concern over eating, weight, and shape significantly decreased during treatment and at follow-up. Furthermore, food restraint behaviours significantly decreased following IBT. It is noteworthy that participants' report of their ideal weight significantly increased from pre- to post-treatment and at follow-up. Thus, they seem to have accepted that a higher weight than which they were aiming for before IBT would be ideal for their body. Additionally, what patients with EDs fear the most at start of therapy, weight 
gain, did not occur in the long term. Although a non-significant increase in median BMI scores was observed following IBT, they reverted back to their initial score at follow-up, which was in the normal range for all participants.

Results revealed a significant decrease in drive for thinness (excessive concern with dieting, preoccupation with weight, and fear of weight gain), bulimia (episodes of binge eating and purging), ineffectiveness (feelings of inadequacy, insecurity, worthlessness and having no control over one's life), perfectionism, difficulty in interoceptive awareness (ability of an individual to discriminate between sensations, feelings, and between the sensations of hunger and satiety), maturity fears, asceticism, difficulty in impulse regulation (ability to regulate impulsive behaviour, especially the binge behaviour), and social insecurity following IBT. A trend was observed towards decrease in body dissatisfaction and interpersonal distrust from pre- to post-IBT, and body dissatisfaction further decreased at follow-up. This suggests that improvements in ED symptoms following IBT extend to areas that are not specifically addressed by IBT such as feelings of ineffectiveness, interoceptive awareness, maturity fears, asceticism, impulse regulation, social insecurity, and interpersonal distrust.

\section{Longitudinal changes in ego-dystonicity and OVI}

In line with our hypothesis, OVI or the degree of conviction towards obsessional doubts decreased significantly over the course of treatment as ED obsessions and compulsions improved. The degree of ego-syntonicity in obsessions and compulsions measured by the YBC-EDS was also found to decrease significantly at six-month follow-up. Furthermore, the irrationality of ED intrusive thoughts was shown to augment over the course of IBT. This is consistent with the decrease in OVI and with IBT's premise that modifying the reasoning narrative producing the obsessional doubt will in turn decrease compulsive behaviour even though treatment was not focused on behaviour change or ERP as in traditional CBT.

\section{Longitudinal changes in cognitive process measures}

Results revealed a significant decrease in participants' fear of who they could become following IBT. Moreover, a significant decrease in inflated responsibility for harm, intolerance of uncertainty/perfectionism, and over-importance/control of thoughts following treatment was observed as well as a trend towards decrease in overestimation of threat scores at follow-up. It is noteworthy that the belief domains of the OBQ changed following IBT although they were not addressed specifically in therapy.

Although a decrease in inferential confusion over the course of therapy was shown, only a significant reduction was found from midto post-IBT. Del Borrello \& O'Connor ${ }^{35}$ demonstrated that changes in levels of inferential confusion were the most predictive of change in OCD symptoms, supporting the theoretical basis of IBT that a decrease in inferential confusion is associated with a decrease in symptoms. This result is also consistent with findings of Paradisis et al., ${ }^{62}$ and Aardema et al., ${ }^{63}$ demonstrating that inferential confusion is a strong predictor of change in OCD symptoms. The current study could not confirm that the decrease in inferential confusion over the course of IBT predicted the improvement in ED symptoms. Nonetheless, in accordance with previous findings, this could be the case, and should be investigated in future research.

\section{Longitudinal changes in depression, anxiety and moti- vational measures}

Depression and anxiety symptoms were both mild at the start of therapy. They improved significantly following IBT. Moreover, the majority of participants reported a change from a feeling of being in the action stage at pre-treatment to a feeling of being in the recovery stage at follow-up. This awareness in the significant progression of participants' motivational stage follows the improvement trajectory in ego-dystonicity in obsessions and compulsions, OVI, fear of self, inferential confusion, obsessional beliefs, ED, depression and anxiety symptoms.

\section{Conclusion}

To conclude, the present study highlights the pertinence to address fear of self and self-doubt in psychological treatment for EDs via a focus on self-cognitions and reasoning about self. Patients can benefit from a therapy exploring other options than constantly running away from their feared identity. Our findings also reveal the importance for future research, so far scarce, on the impact of ego-dystonicity in obsessions and how it relates to an overinvestment in the "self-as could-be", or feared identity, in contrast to the "self-as-is" in EDs. Recent developments have pointed to the importance of examining self-values, sense of self, self-compassion and ambivalence towards the self, and have produced promising interventions. In addition, this study suggests that IBT be considered a promising alternative to traditional CBT especially when OVI are high and underlines the importance of examining the long-term outcome of interventions.

The major limitation of this study is the small sample size, which in turn interferes with the ability to perform more in-depth analyses of predictors and mediators of symptom improvement and change in ego-dystonicity in obsessions and related fear of self. However, the current findings provide good preliminary evidence that these concepts play an important role in BN. Although another limitation is the lack of participants with AN in our sample, the transdiagnostic model of EDs by Fairburn et al., ${ }^{23}$ provides support that all individuals with EDs over-value food/weight/shape thoughts, and use weight control techniques (dieting, restriction) to cope with these thoughts. Therefore, even though some ED symptoms in BN may be distressing, like vomiting, there are a variety of ego-syntonic symptoms (restriction, drive for thinness) that are theoretically expected to be common across diagnoses. We therefore expect IBT's more thorough investigation of cognitive maintaining factors, self-cognitions, reasoning about self and effectiveness in decreasing strong OVI and ego-syntonic obsessions in $\mathrm{BN}$ will extend to treating $\mathrm{AN}$.

Finally, the lack of a control group prevents attributing improvements exclusively to the IBT intervention. Nevertheless, given the exploratory nature of the study and the large effect sizes that were found, the data and conclusions presented here still provide coherent and solid empirical support for the use of IBT in BN. A randomized-controlled trial will be crucial to confirm IBT's efficacy and effectiveness with EDs, and particularly with AN. Further research is needed in order to improve our understanding of ego-dystonicity and ego-syntonicity, fear of self, inferential confusion and contribute to a better comprehension of the variables hindering effective treatment of EDs.

\section{Author's note}

This work was supported by grant number 20573, awarded to the second author, from the Fonds de la recherche en santé du Québec.

\section{Acknowledgments}

None.

\section{Conflicts of interest}

Author declares there are no conflicts of interest. 


\section{Funding}

None.

\section{References}

1. American Psychiatric Association. Diagnostic and Statistical Manual of Mental Disorders, Text Revision (DSM-IV-TR) (4th edn), Washington DC, USA. 2000.

2. Purdon C, Cripps E, Faull M, et al. Development of a Measure of Egodystonicity. Journal of Cognitive Psychotherapy: An International Quarterly. 2007;21(3):198-216.

3. Purcell Lalonde M, O'Connor K, Aardema F, et al. Food for thought: ego-dystonicity and fear of self in eating disorders. Eur Eat Disord Rev. 2014;23(3): 179-184.

4. Foa EB. Failure in treating obsessive-compulsives. Behav Res Ther 1979;17(3):169-176.

5. Neziroglu F, McKay D, Yaryura-Tobias JA, et al. The overvalued ideas scale: Development, reliability and validity in obsessive-compulsive disorder. Behav Res Ther . 1999;37(9):881-902.

6. Christenson DD, Greist JH. The challenge of obsessive-compulsive disorder hoarding. Primary Psychiatry. 2001;8:79-86.

7. Summerfeldt LJ. Incompleteness, ordering and arranging. In: Antony MM, et al. (Eds.), Cognitive behaviour therapy for OCD: Beyond the basics. American Psychological Association, New York, USA. 2006. p.187-208.

8. Roncero M, Belloch A, Perpina C, et al. Ego-syntonicity and egodystonicity of eating-related intrusive thoughts in patients with eating disorders. Psychiatry Res. 2013;208(1):67-73.

9. Mazure CM, Halmi KA, Sunday SR, et al. The Yale-Brown-Cornell Eating Disorder Scale. J Psychiatr Res . 1994;28(5):425-445.

10. Sunday SR, Halmi KA. Comparison of the Yale-Brown-Cornell Eating Disorders Scale in recovered eating disorder patients, restrained dieters, and non-dieting controls. Int J Eat Disord. 2000;28(4):455-459.

11. Clark DA, Purdon C. New perspectives for a cognitive theory of obsessions. Australian Psychologist. 1993;28(3):161-167.

12. Aardema F, O'Connor K. The menace within: Obsessions and self Journal of Cognitive Psychotherapy: An International Quarterly. 2007;21(3):182-197.

13. Aardema F, O'Connor K, Emmelkamp PM, et al. Inferential confusion in obsessive-compulsive disorder: the inferential confusion questionnaire. Behav Res Ther. 2005;43(3):293-308.

14. Rachman S. A cognitive theory of obsessions. Behav Res Ther 1997;35(9):793-802.

15. Markus H, Nurius P. Possible selves. American Psychologist. 1986;41(9):954-969.

16. Ogilvie DM. The undesired self: a neglected variable in personality research. Journal of Personality and Social Psychology. 1987;52(2):379-385.

17. Ferrier S, Brewin CR. Feared identity and obsessive-compulsive disorder. Behav Res Ther. 2005;43(10):1363-1374.

18. Fairburn CG, Cooper Z. The Eating Disorder Examination (12th edn), In: Fairburn CG \& Wilson GT (Eds.), Binge Eating: Nature, assessment, and treatment. Guilford Press, New York, USA. 1993. p.317-360.

19. Leung N, Waller G, Thomas G. Core beliefs in anorexic and bulimic women. J Nerv Ment Dis. 1999;187(12):736-741.

20. Waller G, Ohanian V, Meyer C, et al.Cognitive content among bulimic women: The role of core beliefs. Int J Eat Disord. 2000;28(2):235-241.
21. Purcell Lalonde M, O'Connor K. Cognitive therapy, ego-dystonicity and eating disorders. In: Lee SA \& Edget DM (Eds.), Cognitive Behavioural Therapy: Applications, Methods and Outcomes. Hauppauge, Nova Science Publishers, New York, USA. 2012. p.93-114.

22. Butler AC, Chapman JE, Forman EM, et al. The empirical status of cognitive-behavioral therapy: a review of meta-analyses. Clin Psychol Rev. 2006;26(1):17-31.

23. Fairburn CG, Cooper Z, Shafran R. Cognitive behaviour therapy for eating disorders: A «transdiagnostic» theory and treatment. Behav Res Ther. 2003;41(5):509-528.

24. Eivors A, Button E, Warner S, et al. Understanding the experience of drop-out from treatment for anorexia nervosa. European Eating Disorders Review. 2003;11:90-107.

25. Steketee GS. Treatment of obsessive compulsive disorder. Guilford Press, New York, USA. 1993.

26. Fairburn CG, Beglin SJ. Assessment of eating disorders: Interview or self-report questionnaire? Int J Eat Disord . 1994;16(4):363-370.

27. Shapiro JR, Berkman ND, Brownley KA, et al. Bulimia nervosa treatment: a systematic review of randomized controlled trials. Int $J$ Eat Disord. 2007;40(4):321-336.

28. Steinglass JE, Eisen JL, Attia E, et al. Is anorexia nervosa a delusional disorder? An assessment of eating beliefs in anorexia nervosa. $J$ Psychiatr Pract . 2007;13:65-71.

29. Aardema F, Emmelkamp PMG, O'Connor K. Inferential confusion, cognitive change and treatment outcome in obsessive-compulsive disorder. Clin Psychol Psychother. 2005;12:337-345.

30. Taillon $\mathrm{A}, \mathrm{O}^{\prime} \mathrm{Connor} \mathrm{K}$. Changes in mood and behavior during cognitive therapy for obsessive compulsive disorder with and without overvalued ideation. Paper presented at the Association of Psychological Science Annual Convention, San Francisco, Califonia, USA. 2009.

31. O’Connor K, Koszegi N, Aardema F, et al. An inferenced-based approcah to treating obsessive-compulsive disorder. Cognitive and Behavior Practice. 2009;16:420-429.

32. Taillon A, O'Connor K, Dupuis G, et al. Inference-Based Therapy for Body Dysmorphic Disorder. Clin Psychol Psychother. 2013;20(1):67-76.

33. Bertrand A, O'Connor K (2009) Manuel de traitement des troubles de l'alimentation par une thérapie cognitive: l'approche basée sur les inférences. Unpublished manuscript. Centre de recherche FernandSeguin, Montréal, Canada.

34. O'Connor K, Aardema F, Pelissier MC (2005) Beyond reasonable doubt: reasoning processes in obsessive-compulsive disorder and related disorders. John Wiley \& Sons Ltd., Chichester, UK, pp. 320.

35. Del Borrello L, O'Connor K (2014) The role of obsessive beliefs and inferential confusion in predicting treatment outcomes for different subtypes of obsessive-compulsive disorder. International Journal of Cognitive Therapy 7: 43-66.

36. Attia E, Roberto CA. Should amenorrhea be a diagnostic criterion for anorexia nervosa? Int $J$ Eat Disord. 2009;42(7):581-589.

37. First M, Spitzer R, Gibbon M, et al. Structured clinical interview for DSM-IV axis 1 disorders - Patient edition (SCID-I/P) (2nd edn), New York State Psychiatric Institute: Biometrics Research Department, New York, USA. 1996.

38. Cooper Z, Cooper PJ, Fairburn CG . The validity of the Eating Disorder Examination and its subscales. Br J Psychiatry. 1989;154:807-812.

39. Zanarini MC, Frankenburg FR. Attainment and maintenance of reliability of axis I and axis II disorders over the course of a longitudinal study. Compr Psychiatry. 2001;42(5):369-374.

40. Goodman WK, Price LH, Rasmussen SA, et al. The Yale-Brown Obsessive Compulsive Scale: I. Development, use, and reliability. Arch Gen Psychiatry. 1989;46(11):1006-1011. 
41. Goodman WK, Price LH, Rasmussen SA, et al. The Yale-Brown Obsessive Compulsive Scale: II. Validity. Arch Gen Psychiatry. 1989;46(11):1012-1016.

42. Aardema F, Moulding R, Radomsky AS, et al. Fear of Self in ObsessiveCompulsive Disorder. In K. O'Connor (Chair), When Seeing Is Not Believing: The Role of Doubt in Obsessive Compulsive Disorder. Symposium conducted at the Annual Congress of the European Association for Behavioural and Cognitive Therapies, Marrakech, Morocco. 2013

43. Garner DM. Eating Disorder Inventory-2 Manual. Psychological Assessment Resources, Odessa, Florida, USA. 1991.

44. Garner DM, Olmsted MA. Eating disorder manual. Lutz, Florida: Psychological Assessment Resources, Inc. 1984.

45. Welch G. Selected multivariate statistical techniques and eating disorders. University of Otago, New Zealand. 1988.

46. DiClemente C, Prochaska JO. Towards a comprehensive, transtheorectical model of change. In: Miller W \& Heather N (Eds.) Treating Addictive Behaviours. Plenum Press, New York, USA. 1998. p.3-24.

47. Gusella J, Butler G, Nichols L, et al. A brief questionnaire to assess readiness to change in adolescents with eating disorders: Its application to group therapy. European Eating Disorders Review. 2003;11(1):58-71.

48. Beck AT, Epstein N, Brown G, et al. An inventory for measuring clinical anxiety: Psychometric properties. J Consult Clin Psychol. 1988;56(6):893-897.

49. Freeston $\mathrm{MH}$, Rhéaume J, Letarte $\mathrm{H}$, et al. Why do people worry? Personality and Individual Differences. 1994;17:791-802.

50. Beck AT, Steer RA, Brown G. Manual for Beck Depression Inventory II. Psychological Corporation, San Antonio, Texas, USA. 1996.

51. Harcourt Brace. Company. Canada. 1998.

52. Hyler SE. PDQ-4 and PDQ-4+ instructions for use. New York State Psychiatric Institute, New York, USA. 1997.

53. Obsessive Compulsive Cognitions Working Group. Psychometric validation of the obsessive belief questionnaire and interpretation of intrusions inventory-- Part 2: Factor analyses and testing of a brief version. Behav Res Ther. 2005;43(11):1527-1542.
54. Julien D, Careau Y, O'Connor KP, et al. Specificity of belief domains in OCD: Validation of the French version of the Obssessive Beliefs Questionnaire and a comparison across samples. J Anxiety Disord. 2008;22(6):1029-1041.

55. Aardema F, Wu K, Careau Y, et al. The expanded version of the inferential confusion questionnaire: Further development and validation in non-clinical and clinical samples. Journal of Psychopathology \& Behavioral Assessment. 2010;32:448-462.

56. Fairburn CG. Cognitive behavior therapy and eating disorders. New York, USA. 2008

57. Keys A, Brozek J, Henschel A, et al. The biology of human starvation. University of Minnesota Press, Minneapolis, USA. 1950.

58. Tabachnick BG, Fidell LS. Using Multivariate Analysis (4th edn). Boston. 2001

59. Cohen J. Statistical power analysis for the behavioural sciences (2nd edn), Academic Press, New York, USA. 1988.

60. Emmelkamp PMG. Commentary on treatment. In: Frost RO \& Steketee $\mathrm{G}$ (Eds.), Cognitive approaches to obsessions and compulsions: Theory, assessment, and treatment. Oxford, Elsevier, UK. 2002. p.391-401.

61. Fairburn CG, Cooper Z, Shafran R. Cognitive behaviour therapy for eating disorders: A «transdiagnostic» theory and treatment. Behav Res Ther. 2003;41(5):509-528.

62. Paradisis SM, Aardema F, O’Connor K. Imaginative, Schizotypal, and Dissociative Processes Predicting OCD Treatment Outcome. In: O'Connor $\mathrm{K}$ (Chair), Believed-In Imaginings in OCD: New Experimental and Clinical Findings. Symposium conducted at the Annual Congress of the Association for Behavioral and Cognitive Therapies, Toronto, Canada. 2011.

63. Aardema F, Radomsky AS, O'Connor KP,et al. Inferential confusion, obsessive beliefs and obsessive-compulsive symptoms: A multidimensional investigation of cognitive domains. Clinical Psychol Psychother. 2008;15(4):227-238. 